\title{
openheart Diagnostic accuracies of screening for atrial fibrillation by cardiac nurses versus radiographers
}

\author{
Lærke Marius Kvist, ${ }^{\oplus 1}$ Nicklas Vinter, ${ }^{1,2}$ Grazina Urbonaviciene, ${ }^{1,2}$ \\ Jes Sanddal Lindholt, ${ }^{3}$ Axel Cosmus Pyndt Diederichsen, ${ }^{\circ}{ }^{4}$ Lars Frost ${ }^{5}$
}

To cite: Kvist LM, Vinter N, Urbonaviciene $\mathrm{G}$, et al. Diagnostic accuracies of screening for atrial fibrillation by cardiac nurses versus radiographers. Open Heart 2019;6:e000942. doi:10.1136/ openhrt-2018-000942

Received 1 October 2018 Revised 11 December 2018 Accepted 3 February 2019

Check for updates

(c) Author(s) (or their employer(s)) 2019. Re-use permitted under CC BY-NC. No commercial re-use. See rights and permissions. Published by BMJ.

${ }^{1}$ Diagnostic Centre, Regionshospitalet Silkeborg, Silkeborg, Denmark ${ }^{2}$ Department of Clinical Medicine, Aarhus Universitet, Aarhus, Denmark ${ }^{3}$ Department of Cardiothoracic and Vascular Surgery, Elitary Research Centre of Individualized Medicine in Arterial Diseases (CIMA), Odense Universitetshospital, Odense, Denmark

${ }^{4}$ Department of Cardiology, Odense University Hospital, Odense, Denmark

${ }^{5}$ Department of Medicine, Silkeborg Regional Hospital and Cardiovascular Research Centre Viborg and Silkeborg Hospital and Institute of Clinical Medicine, Aarhus University Hospital Denmark, Silkeborg, Denmark

Correspondence to Dr Lærke Marius Kvist; mariuskvist@gmail.com

\section{ABSTRACT}

Aim We examined the diagnostic accuracy of single-lead ECG as assessed by radiographers and 12-lead ECG as assessed by cardiac nurses for the diagnosis of atrial fibrillation (AF).

Methods Based on the Danish Cardiovascular Screening Trial, we conducted a population-based, cross-sectional study of 1338 randomly selected Danish men aged 65-74 years with no exclusion criteria. The participants were screened with single-lead ECG during a CT scan assessed by radiographers and 12-lead ECG assessed by cardiac nurses. The diagnostic accuracy was evaluated compared with that produced by a 12-lead ECG assessed by two consenting cardiologists.

Results The study identified 68 participants with ongoing AF, of whom 60 had self-reported AF and 8 had AF detected in the screening. Single-lead ECG assessed for AF by radiographers had a sensitivity of $60.3 \%(95 \% \mathrm{Cl}$ 47.7 to 72.0$)$, specificity of $97.2 \%(95 \% \mathrm{Cl} 96.2$ to 98.1$)$, positive predictive value (PPV) of $53.9 \%(95 \% \mathrm{Cl} 42.1$ to 65.5 ) and negative predictive value (NPV) of $97.9 \%$ (95\% Cl 96.9 to 98.6). 12-lead ECG assessed by cardiac nurses had a sensitivity of $97.1 \%$ (95\% Cl 89.8 to 99.6$)$, specificity of $100 \%$ (95\% Cl 99.7 to 100 ), PPV of $100 \%$ (95\% Cl 94.6 to 100$)$ and NPV of $99.8 \%$ (95\% Cl 99.4 to 100).

Conclusions Single-lead ECG assessed by radiographers had a moderate sensitivity and PPV but a very high specificity and NPV. Using radiographers may be acceptable for opportunistic screening, in particular if radiographers are thoroughly trained. Thus, 12-lead ECG assessed by cardiac nurses is a potential diagnostic method for the detection of AF.

\section{INTRODUCTION}

Atrial fibrillation (AF) is one of the most common arrhythmias, with an increasing incidence and prevalence in developed countries, especially among men and the elderly. ${ }^{1}$ In the European Union, the prevalence of $\mathrm{AF}$ is estimated to double from 8.8 million in 2010 to 17.9 million in $2060 .^{2}$

Up to one-third of patients with AF are asymptomatic, whereas others may present with symptoms or AF-related sequelae such as ischaemic stroke. ${ }^{3} 4$ Patients with

\section{Key questions}

What is already known about this subject?

- Numerous studies have demonstrated the potential usefulness of a variety of devices such as automated blood pressure measurement, smartphone-based photoplethysmography, handheld single-lead ECG and other non-12-lead ECG devices for the diagnosis of atrial fibrillation (AF); these devices are potential screening tools for AF.

What does this study add?

- Our study adds to the knowledge of the diagnostic accuracy of single-lead and 12-lead ECG assessed by non-specialists for the diagnosis of $A F$.

How might this impact on clinical practice?

- Single-lead ECG assessed by appropriately trained radiographers during a CT scan could potentially be used for opportunistic screening for AF.

- A 12-lead ECG assessed by cardiac nurses may be useful for the diagnosis of AF.

asymptomatic AF have a significantly higher risk of cardiovascular and all-cause mortality compared with patients with symptomatic $\mathrm{AF}^{5} \mathrm{AF}$ is a major cause of stroke, and previously unknown AF is associated with approximately $10 \%$ of all ischaemic strokes, causing both severe morbidity and mortality. ${ }^{3}$ Consequently, screening for AF is debated worldwide. $^{346}$ The STROKESTOP study reported that among patients with known AF, only $78 \%$ were treated with oral anticoagulation (OAC) at the time of screening. ${ }^{7}$ Other studies have shown a similar lack of or a suboptimal treatment with OAC. ${ }^{8}$ As OAC treatment may reduce the risk of stroke by more than $60 \%,{ }^{9}$ the identification of patients with untreated or unknown AF is needed. ${ }^{349}$

The gold standard for the diagnosis of AF is 12-lead ECG. ${ }^{9}$ During recent years, numerous studies have demonstrated the potential usefulness of other non-12-lead ECG devices. ${ }^{40-13}$ In the SAFE study, opportunistic screening by pulse palpation was found to be 
superior to both routine care and systematic screening in identifying new cases of $\mathrm{AF},{ }^{14}$ and other modalities may have a higher accuracy than pulse palpation. ${ }^{15}$ The European Society of Cardiology (ESC) guidelines recommend opportunistic screening for AF by pulse palpation or the use of cardiac rhythm recording in patients $>65$ years of age; in cases of an irregular pulse, a 12-lead ECG should be recorded to confirm $\mathrm{AF}^{9}{ }^{9}$ However, there is not yet definite evidence that screening for $\mathrm{AF}$ improves outcomes. ${ }^{3}$

The Danish Cardiovascular Screening Trial (DANCAVAS) is a randomised outcome trial measuring whether systematic multifaceted screening for cardiovascular disease impacts total mortality. ${ }^{16}$ In this substudy of the DANCAVAS Trial, we aimed to examine the diagnostic accuracy of CT-related single-lead ECG assessed by radiographers (radiograph-CT-ECG) as an opportunistic screening tool and 12-lead ECG assessed by cardiac nurses (nurse-ECG) for the diagnosis of AF (and flutter). We further aimed to evaluate the acceptability of OAC among participants who screened positive for unknown $\mathrm{AF}$.

\section{METHODS}

\section{Study design}

This is a population-based, cross-sectional diagnostic study in which the participants underwent cardiovascular screening. All data were prospectively collected.

\section{Participants}

The study cohort is based on the DANCAVAS Trial, the protocol of which is accessible elsewhere. ${ }^{16}$ Briefly, 45 000 Danish men aged between 65 and 74 years were randomised to participate in the screening programme. A random third of the 45000 men were invited to the screening programme, and the remaining individuals were used as the control group. No exclusion criteria were applied because the intention of the DANCAVAS Trial was to increase generalisability. ${ }^{16}$ Of the 45000 men, 2361 were invited to screening at the Silkeborg Regional Hospital. The Civil Registration System provided information on the civil registration number, name and address. The screening modalities used in the DANCAVAS Trial are described in detail in the protocol, and all participants were screened by radiograph-CT-ECG. ${ }^{16}$

After the pilot phase of the DANCAVAS Trial comprising 331 participants, a standard 12-lead ECG was additionally introduced to the screening programme at the Silkeborg Regional Hospital. All participants were randomly invited to screening from the general population. Our substudy was conducted from February 2016 to February 2018.

The tests performed during this study had no adverse effects, and the use of 12-lead ECG is thought to be safe. The non-contrast CT scan exposed the participants to a median radiation of 3.6 (IQR 2.9-4.7) milliSievert (mSv). In comparison, the average annual worldwide exposure to radiation is $2.4 \mathrm{mSv} .{ }^{17} \mathrm{~A}$ CT scan therefore constitutes a minor additional risk of developing adverse effects such as radiation-related cancers.

\section{Test methods}

All participants reported medical history, including history of previously diagnosed AF and comorbidities, and the use of medication. In the DANCAVAS Trial, the participants were screened for coronary artery calcifications and aortic/iliac aneurysms by CT scan. The CT scans were performed with a 320-slice volume CT scanner (Aquilion One, Toshiba Medical Systems, Japan). As the CT scan uses single-lead ECG (extremity lead I) to trigger the processing of the images during diastole, the radiographers were allowed to screen for AF. The average duration of a single-lead ECG recording was 5-10 min. The single-lead ECG recordings could not be stored for later re-evaluation.

During the study period, one of eight alternating radiographers examined each single-lead ECG for AF. The radiographers had oral and written training in ECG assessment with a focus on the ECG characteristics of AF. A research nurse trained in cardiology was responsible for the training. The training session consisted of a thorough introduction to the normal ECG, and subsequently an electrocardiographic description of cardiac arrhythmias with emphasis on $\mathrm{AF}$, in particular the identification of no distinct $P$ waves and irregular RR intervals. Furthermore, the training included case-based exercises. During the first 2 weeks of the study, the radiographers had access to supervision by cardiac nurses. The written training material was available for the radiographers throughout the entire screening period.

Within a maximum of 1 hour after the CT scan, the participants had a 12-lead ECG recorded (Schiller Cardiovit AT-102, Schiller Cardiovit AT-102 Plus or Philips PageWriter Trim II). The 12-lead ECGs were examined for AF by one of four study nurses. All of the four nurses had training in ECG and experience with patients with $\mathrm{AF}$ from working at a cardiology ward for 4-20 years. The nurses had no access to the radiographer's interpretations of the single-lead ECGs, but they did have knowledge about the participant-reported medical history and medication. Finally, two independent cardiologists examined all of the 12-lead ECG recordings, which we used as the reference standard for the verification of AF. In the case of any disagreements, a consensus was made between the two cardiologists. The cardiologists had no knowledge of the related medical history and the use of medications, and the cardiologists were blinded to the reports from both the radiographers and the nurses.

If a diagnosis of AF was made by 12-lead ECG assessed by a cardiologist, the participant was invited for follow-up in the outpatient clinic to assess the risk for thromboembolism using the $\mathrm{CHA}_{2} \mathrm{DS}_{2}$-VASc (congestive heart failure, hypertension, age $>75$ years, diabetes mellitus, stroke/transient ischemic attack, vascular disease, age 65-74 years, sex category) score. ${ }^{9}$ The need for treatment in accordance with the ESC guidelines was evaluated in 
cases of both newly diagnosed $\mathrm{AF}$ and self-reported $\mathrm{AF}$ without sufficient OAC treatment.

\section{Statistics}

Categorical variables were presented as total numbers and percentages and continuous variables as medians with IQR. The prevalence of AF among the screened population was presented as the total numbers and percentages, with a 95\% CI. The distribution of participants with self-reported and unknown $\mathrm{AF}$ was presented as the total numbers and percentages. The sensitivity, specificity, and negative and positive predictive values (NPV and PPV), including 95\% CI, for radiograph-CT-ECG and nurse-ECG compared with 12-lead ECG assessed by cardiologists were calculated. The $95 \%$ CIs were calculated as the exact Clopper-Pearson interval. Furthermore, the distribution of initiated OAC treatment among participants with self-reported and new AF was evaluated and presented as the total numbers and percentages. Data were analysed using Stata V.14.2.

\section{RESULTS}

\section{Characteristics of participants}

Of the 2361 randomly selected men who were invited to be screened at the Silkeborg Regional Hospital, 1671 accepted the invitation (70.8\%). As the standard 12-lead ECG was introduced after a pilot phase comprising 331 participants and 2 participants left the screening location before the 12-lead ECG was recorded, our study

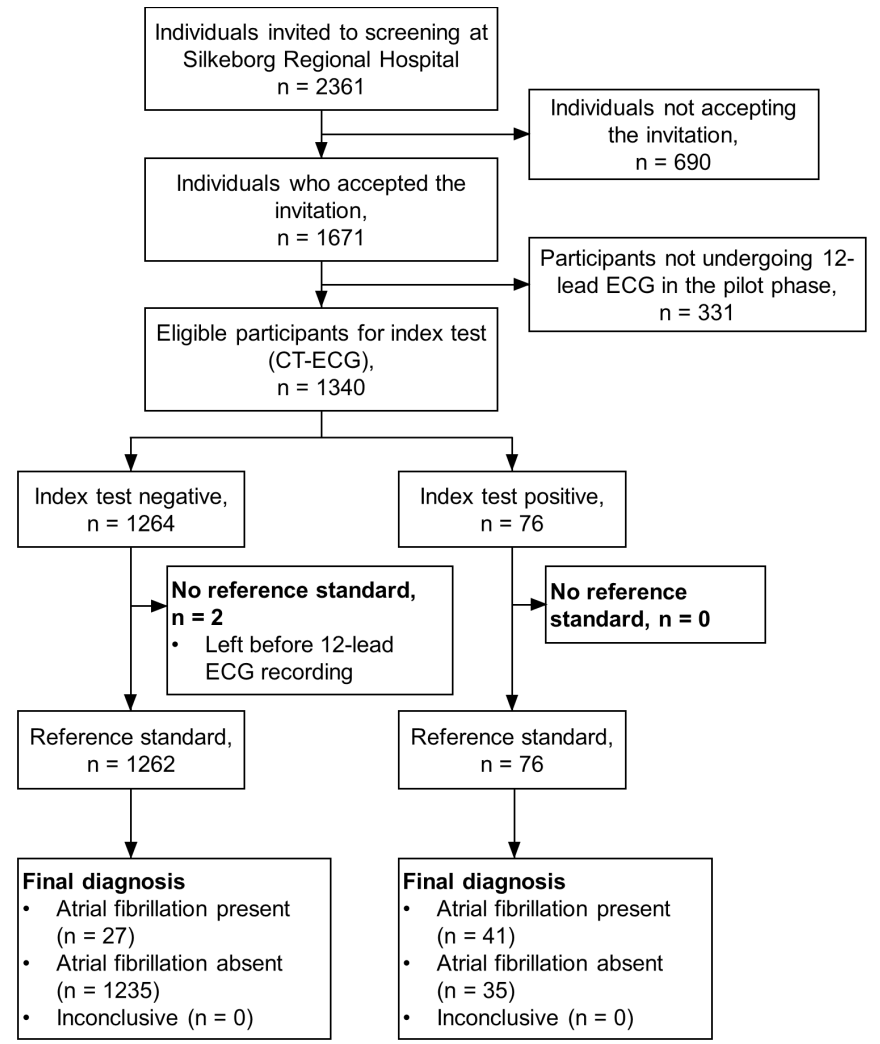

Figure 1 Flow chart. The figure shows the index test for the radiographers only. population consisted of $1338 \quad(56.7 \%)$ participants (figure 1).

The clinical characteristics of the 1338 participants who underwent both CT-ECG and 12-lead ECG are shown in table 1 . The median age was 69 years, and the most frequent comorbidities were hypertension $(42.4 \%)$ and diabetes $(10.9 \%)$.

\section{Prevalence and distribution of $\mathrm{AF}$}

A previous diagnosis of $\mathrm{AF}$ was reported by $7.9 \%$ $(n=106 / 1338)$ of the participants. Information on self-reported AF, previously confirmed to the participant by a cardiologist or a general practitioner (GP), was missing for four participants $(0.3 \%)$, of whom none were diagnosed with $\mathrm{AF}$ at the time of the screening. Of the 106 participants with self-reported AF, 60 (56.6\%) had ongoing AF on 12-lead ECG assessed by cardiologists. Of the 1228 participants without self-reported AF, 0.7\% (95\% CI 0.03 to 1.3$) \quad(n=8 / 1228)$ were diagnosed with new AF on 12-lead ECG assessed by cardiologists. The prevalence of ongoing AF detected by 12-lead ECG was $5.1 \%(95 \%$ CI 4.0 to 6.4$)(\mathrm{n}=68 / 1338)$. The prevalence of $\mathrm{AF}$ in the screened population was $8.5 \%$ (95\% CI 7.1 to 10.0$)(n=114 / 1338)$. Atrial flutter was present in $4.4 \%$ $(n=3 / 68)$ of the cases with ongoing AF.

\section{Diagnostic performances}

The results from the recordings by radiograph-CT-ECG and nurse-ECG are listed in table 2, and the results of the diagnostic performances are listed in table 3. Radiograph-CT-ECG correctly identified 41 patients as having $\mathrm{AF}$ and misdiagnosed 27 patients (39.7\%). Nurse-ECG correctly identified 66 patients as having AF and misdiagnosed 2 patients $(2.9 \%)$. The accuracy of the radiograph-CT-ECG was $95.4 \%$ (95\% CI 94.1 to 96.4), due to 35 false positive and 27 false negative diagnoses. The accuracy of the nurse-ECG was $99.9 \%$ (95\% CI 99.4 to 100 ), due to 0 false positive and 2 false negative diagnoses.

\section{OAC treatment}

All participants with a new diagnosis of AF or self-reported $\mathrm{AF}$ who were not on $\mathrm{OAC}$ treatment attended the follow-up in the outpatient clinic. Patients with AF aged 65 years or above have a $\mathrm{CHA}_{2} \mathrm{DS}_{2}$-VASc score of at least 1 . Therefore, participants with AF in our study were candidates for OAC therapy according to clinical guidelines, independent of other risk factors for stroke. ${ }^{9}$ Among the 60 participants with previously diagnosed AF, 56 (93.3\%) were in relevant OAC treatment. Of the remaining 4 cases, $1(1.7 \%)$ initiated OAC treatment and $2(3.3 \%)$ chose not to undergo treatment due to a $\mathrm{CHA}_{2} \mathrm{DS}_{2}$-VASc score of 1 . In the remaining case $(1.7 \%)$, treatment was considered to be contraindicated due to excessive alcohol abuse. Of the 8 participants with previously unknown AF, 2 (25.0\%) were already treated with OAC due to a mechanical heart valve. The remaining $6(75.0 \%)$ initiated OAC treatment due to a $\mathrm{CHA}_{2} \mathrm{DS}_{2}$-VASc score of 1 or above. 
Table 1 Self-reported clinical characteristics of participants prior to screening

\begin{tabular}{|c|c|}
\hline Clinical characteristics & $\begin{array}{l}\text { Participants, } \\
\mathrm{N}=1338\end{array}$ \\
\hline Age, median (IQR), years & $69.0(67.0-71.0)$ \\
\hline Body mass index, median (IQR), $\mathrm{kg} / \mathrm{m}^{2}$ & $27.3(25.0-30.3)$ \\
\hline \multicolumn{2}{|l|}{ Self-reported conditions, $n$ (\%) } \\
\hline $\mathrm{AF}^{*}$ & $106(7.9)$ \\
\hline Diabetes & $146(10.9)$ \\
\hline Hypertension & 567 (42.4) \\
\hline Ischaemic stroke & $81(6.1)$ \\
\hline Acute myocardial infarction & $83(6.2)$ \\
\hline Peripheral arterial disease & $30(2.2)$ \\
\hline $\mathrm{CABG}$ or $\mathrm{PCl}$ & $11(8.3)$ \\
\hline Chronic obstructive pulmonary disease & $91(6.8)$ \\
\hline \multicolumn{2}{|l|}{ Smoking, n (\%) } \\
\hline Never & 451 (33.9) \\
\hline Former & $687(51.6)$ \\
\hline Current & $193(14.5)$ \\
\hline \multicolumn{2}{|l|}{ Medication, n (\%) } \\
\hline Antiplatelets & $318(23.8)$ \\
\hline OAC for any indication $†$ & $114(8.5)$ \\
\hline Statins & $476(35.6)$ \\
\hline Antiarrhythmic drug & $14(1.1)$ \\
\hline Thiazide & $55(4.1)$ \\
\hline Beta-antagonists & 207 (15.5) \\
\hline ACE inhibitor/ARB & $484(36.2)$ \\
\hline $\mathrm{Ca}^{++}$antagonist & $265(19.8)$ \\
\hline Potassium-sparing diuretics & $79(5.9)$ \\
\hline
\end{tabular}

Missing data, $\mathrm{n}(\%)$ : body mass index 1 (0.07), AF $4(0.3)$ and smoking 7 (0.5).

*As informed by the general practitioner or during hospitalisation. †The use of warfarin, novel OACs or phenprocoumon for any indication, for example, AF, venous thromboembolism or mechanical heart valves.

$A F$, atrial fibrillation; ARB, angiotensin II receptor blockers; CABG, coronary artery bypass grafting; $\mathrm{OAC}$, oral anticoagulation; $\mathrm{PCI}$, percutaneous coronary intervention.

\section{DISCUSSION}

\section{Principal findings}

In this population-based, cross-sectional diagnostic study among men aged 65-74 years, we demonstrated a moderate diagnostic performance of radiograph-CT-ECG for the diagnosis of AF. This may be an acceptable method for opportunistic screening. Thus, 12-lead-ECG assessed by cardiac nurses may be a potential diagnostic method for $\mathrm{AF}$ due to its very high diagnostic performance.

\section{Diagnostic performances}

In our study, radiograph-CT-ECG identified $60.3 \%$ of participants with ongoing $\mathrm{AF}$, and in the case of a positive test result the probability of a correct diagnosis was
$53.9 \%$. The test performed well at excluding AF (specificity and NPV $>97 \%$ ). This finding indicates a moderate performance of radiograph-CT-ECG for the diagnosis of AF. The diagnostic performances of single-lead ECG without software-based algorithms for detection of AF reported by other studies are shown in table 4 . The SAFE study evaluated opportunistic and systematic screening for AF versus routine care in general practice. ${ }^{14}$ The study included both women and men $>65$ years of age (median age of 74.1 years). Single-lead ECGs collected from both screening arms were assessed by both GPs and practice nurses compared with 12-lead ECG as assessed by cardiologists. Approximately 50\% of the nurses and GPs had training in the interpretation of ECGs, but the extent and kind of the training were not further described. ${ }^{14}$ The SAFE (Screening for Atrial Fibrillation in the Elderly) study had a better diagnostic ability to identify AF by single-lead ECG among GPs compared with practice nurses, probably due to the better education among the GPs. The performance of the practice nurses in the mentioned study was similar to that of radiograph-CT-ECG in our study, which indicates potential room for educational improvements. However, the predictive values may not be perfectly comparable with those obtained by our study, as the SAFE study reported an estimated prevalence of $6.9 \%$ in both screening arms, as verified by the evidence of $\mathrm{AF}$ in the medical records. ${ }^{14}$

Our study showed that 12-lead ECG read by cardiac nurses had a very high diagnostic performance. The SAFE study also examined the diagnostic performance of a 12-lead ECG assessed by practice nurses and GPs (table 4). ${ }^{14}$ However, the discrepancies in diagnostic performance between our study and the SAFE study may be explained by a greater ability among nurses with up to 20 years of experience in cardiology to assess 12-lead ECGs compared with both practice nurses and GPs. In our study, 4 cardiac nurses assessed all ECGs compared with the 22 nurses and GPs in the SAFE study. ${ }^{14}$

An opportunistic screening study for AF in men and women $>65$ years of age (mean age 76 ) entering pharmacies in Sydney, Australia, reported the diagnostic performance of single-lead ECG assessed by pharmacists (table 4) ${ }^{18}$ The pharmacists' interpretation of singlelead ECG was compared with that of single-lead ECG assessed by a cardiologist. Predictive values were not reported. The prevalence of ongoing screen-detected $\mathrm{AF}$ in the mentioned study was $6.7 \%$ compared with $5.1 \%$ in our study. However, the results of our study are not directly comparable due to a highly selected study population in the mentioned study, ${ }^{18}$ superimposed by the inconsistent use of a gold standard as the reference standard. Furthermore, the pharmacists performed pulse palpation and recorded a brief medical history for the participants, which may have influenced their interpretation of the single-lead ECG and led to clinical review bias. 
Table 2 Test results from the index tests and reference standard

\begin{tabular}{|c|c|c|c|}
\hline & \multicolumn{3}{|c|}{ Reference standard (12-lead ECG, cardiologist) } \\
\hline & Positive AF & Negative AF & Total \\
\hline \multicolumn{4}{|c|}{ Index test (radiograph-CT-ECG) } \\
\hline Negative AF & 27 & 1235 & 1262 \\
\hline Total & 68 & 1270 & 1338 \\
\hline Positive AF & 66 & 0 & 66 \\
\hline Negative AF & 2 & 1270 & 1272 \\
\hline Total & 68 & 1270 & 1338 \\
\hline
\end{tabular}

AF, atrial fibrillation; nurse-ECG, 12-lead ECG assessed by nurses; radiograph-CT-ECG, CT-related single-lead ECG assessed by radiographers.

\section{Strengths and weaknesses}

In the DANCAVAS Trial, 2361 men were invited to Silkeborg Regional Hospital, of whom 690 (29.2\%) did not accept the invitation. As participation in the trial was voluntarily, a self-selection bias is likely. We do not expect that the 331 participants only undergoing 12-lead ECG were different from participants not undergoing 12-lead ECG because the order of recruitment was random.

Because our study was a population-based study with no exclusion criteria, our finding may be generalisable to the elderly, mainly Caucasian men of Nordic and European descent. However, our findings cannot be extended to women, other age categories and other races or populations with a substantially different risk profile. Furthermore, the nurses in our study had up to 20 years of experience working at a cardiology ward; therefore, our finding cannot be generalised to all nurses. Other studies have shown a lower diagnostic performance among nurses working in the general practice setting. ${ }^{15}$

Only two ECG recordings were missed, and assessments for AF by cardiologists, nurses and radiographers were available for all 1338 of the included participants. Because the CT scan used in our study could not save the recordings of single-lead ECG, we were not able to perform an evaluation of the diagnostic performance of single-lead ECG assessed by cardiac nurses or cardiologists. The moderate diagnostic performance by radiograph-CT-ECG may be a result of the inadequate training of the radiographers or due to a lower ability of single-lead ECG to diagnose AF. Furthermore, as the nurses had knowledge of related medical history and use of medication among the participants, there is a risk of clinical review bias with a tendency towards a better diagnostic performance of nurse-ECG.

Our study identified $5.2 \%$ patients with AF using 12-lead ECG compared with $7.9 \%$ of participants with self-reported AF. This result points to the weakness of single time-point screening to identify participants with paroxysmal and persistent AF, who may be in normal sinus rhythm (SR) at the time of screening. Furthermore, there is a risk of change in heart rhythm between $\mathrm{AF}$ and normal SR in between CT-ECG and 12-lead ECG.

\section{Implications}

Our study demonstrates that 12-lead ECG as assessed by cardiac nurses may be a potential diagnostic method for the detection of AF. Furthermore, findings from our study and the results reported by the SAFE study ${ }^{14}$ and Lowres et $a l^{18}$ demonstrate that single-lead ECG assessed by non-specialists is not recommended as a diagnostic tool for AF. However, the use of single-lead ECG during a CT scan is a potential method for opportunistic screening. Neither systematic mass screening nor widespread opportunistic screening using 12-lead ECG seems as feasible as the use of single-lead ECG devices. The diagnostic performance of radiograph-CT-ECG for opportunistic screening seems acceptable compared with the alternative of not being diagnosed. It seems likely that the performance of radiograph-CT-ECG could be improved by better training. We urgently need evidence

Table 3 Diagnostic performances of single-lead ECG and 12-lead ECG

\begin{tabular}{|c|c|c|c|c|}
\hline Index text & $\begin{array}{l}\text { Sensitivity } \\
\%(95 \% \text { Cl) }\end{array}$ & $\begin{array}{l}\text { Specificity } \\
\%(95 \% \text { Cl) }\end{array}$ & $\begin{array}{l}\text { PPV } \\
\%(95 \% \mathrm{Cl})\end{array}$ & $\begin{array}{l}\text { NPV } \\
\%(95 \% \mathrm{Cl})\end{array}$ \\
\hline Radiograph-CT-ECG & 60.3 (47.7 to 72.0 ) & 97.2 (96.2 to 98.1) & 53.9 (42.1 to 65.5$)$ & 97.9 (96.9 to 98.6$)$ \\
\hline Nurse-ECG & 97.1 (89.8 to 99.6$)$ & 100 (99.7 to 100$)$ & 100 (94.6 to 100) & 99.8 (99.4 to 100$)$ \\
\hline
\end{tabular}

NPV, negative predictive value; PPV, positive predictive value; nurse-ECG, 12-lead ECG assessed by nurses; radiograph-CT-ECG, CT-related single-lead ECG assessed by radiographers. 


\begin{tabular}{|c|c|c|c|c|c|c|}
\hline Study & Method & $\begin{array}{l}\text { Assessed } \\
\text { by }\end{array}$ & $\begin{array}{l}\text { Sensitivity } \\
\%(95 \% \mathrm{Cl})\end{array}$ & $\begin{array}{l}\text { Specificity } \\
\%(95 \% \text { Cl) }\end{array}$ & $\begin{array}{l}\text { PPV } \\
\%(95 \% \mathrm{CI})\end{array}$ & $\begin{array}{l}\text { NPV } \\
\%(95 \% \mathrm{Cl})\end{array}$ \\
\hline \multirow{2}{*}{$\begin{array}{l}\text { The SAFE } \\
\text { study, } \\
\text { Hobbs et } \\
\text { al }^{14}\end{array}$} & \multirow{2}{*}{$\begin{array}{l}\text { Multicentre RCT. } \\
\text { Opportunistic screening in } \\
\text { primary care or systematic } \\
\text { screening for AF. }\end{array}$} & GPS & 85.4 (78.5 to 90.5$)$ & 86.4 (84.4 to 88.1$)$ & 38.4 (33.0 to 44.1$)$ & 98.4 (97.5 to 99.0$)$ \\
\hline & & Practice nurses & 68.7 (60.4 to 75.9$)$ & 82.7 (80.5 to 84.7 ) & 29.3 (24.5 to 34.6 ) & 96.2 (94.9 to 97.2 ) \\
\hline $\begin{array}{l}\text { Lowres } \\
\text { et } a l^{18}\end{array}$ & $\begin{array}{l}\text { Community-based, cross- } \\
\text { sectional study. } \\
\text { Opportunistic screening for } \\
\text { AF in pharmacies. }\end{array}$ & Pharmacists & 77 (65 to 87) & 87 (85 to 89) & NA & NA \\
\hline
\end{tabular}

AF, atrial fibrillation; GPs, general practitioners; NA, not available; NPV, negative predictive value; PPV, positive predictive value; RCT, randomised controlled trial; SAFE, Screening for Atrial Fibrillation in the Elderly .

about how to educate and reinforce non-specialists to assess single-lead ECGs for the presence or absence of AF. Supervised training, web-based training, certification and audits could be potential tools. Furthermore, the application of an automated algorithm have shown a better diagnostic performance of single-lead ECG for the diagnosis of $\mathrm{AF}^{3}{ }^{3619}$ Future studies are recommended to validate the potentially better diagnostic performance of single-lead ECG assessed by adequately trained medical staff. However, we fully agree with the recent European Heart Rhythm Association position paper that, given the current evidence, OAC treatment should be reserved to patients with 12-lead ECG-confirmed or Holter-confirmed $\mathrm{AF}$ and a $\mathrm{CHA}_{2} \mathrm{DS}_{2}$-VASc score of at least 1 among men and 2 among women. ${ }^{6}$

By screening for $\mathrm{AF}$ above the age of 74 years, the likelihood of identifying AF at single time-point screening increases, as progression from paroxysmal to permanent $\mathrm{AF}$ increases with age. ${ }^{20}$ Additionally, by performing prolonged or intermittent ECG monitoring, the probability of identifying additional AF cases most likely increases, although the prognostic value of intervention in external device-detected AF still needs to be proven. ${ }^{6} 2122$ In patients with cardiac implantable electronic devices, such as pacemakers, implantable cardioverter defibrillators and implantable loop recorders, or in patients undergoing cardiac resynchronisation therapy, the occurrence of atrial high rate episodes (AHRE) has been reported. ${ }^{23}$ AHRE seems to be associated with an increased risk of stroke. ${ }^{24}$ However, there is currently no evidence supporting the need for treatment among those patients, and studies randomising patients with device-detected AHREs to OAC treatment are currently recruiting participants. ${ }^{25} 26$

In the DANCAVAS Trial, the participants are screened for multiple cardiovascular diseases, such as AF, peripheral arterial disease, coronary artery calcifications, aneurysms and traditional risk factors. ${ }^{16}$ The approach of screening for a broad spectrum of diseases with common risk factors may be more cost-effective, and screening for AF may be incorporated into screening programmes for cardiovascular diseases. A randomised multifaceted screening trial for vascular disease, the
Viborg Vascular Trial (VIVA), reported a significant reduction in the risk of mortality among men in the screening arm, mainly due to the initiation of pharmacological therapy. ${ }^{27}$ However, the VIVA study did not include screening for AF.

\section{CONCLUSION}

Single-lead ECG assessed by radiographers for the diagnosis of $\mathrm{AF}$ is not recommended as a diagnostic tool due to its moderate sensitivity and PPV. However, single-lead ECG during a CT scan may be acceptable for opportunistic screening for $\mathrm{AF}$ if the radiographers are thoroughly educated. Thus, 12-lead ECG assessed by cardiac nurses had a very high sensitivity, specificity, PPV and NPV and may be a potential method for the diagnosis of AF.

Acknowledgements We thank all the radiographers and project nurses working at the DANCAVAS study for the collection of data, and the DANCAVAS Coordinating Centre of Odense University Hospital, Denmark.

Contributors GU, JSL, ACD and LF participated in the original planning, conduct and design of the study. LMK, NV and LF performed the data analysis. LMK, NV, GU, JSL, ACD and LF participated in the interpretation of data, as well as the drafting and critical review of the paper. LMK and LF take responsibility for the overall content as guarantors.

Funding This work was supported by the Region of Southern Denmark, the Danish Heart Foundation, the Elitary Research Centre of Individualized Medicine in Arterial Disease (CIMA), the Odense University Hospital, and the Free National Research Councils and Helsefonden. The CT scan and room facilities were provided by the Silkeborg Regional Hospital.

Competing interests LF has been a member of the advisory boards for BMS and Pfizer, and has received speaker fees from Bayer, BMS, Boehringer Ingelheim, MSD and Pfizer.

Patient consent for publication Not required.

Ethics approval The study was approved by the Southern Denmark Region Committee on Biomedical Research Ethics (S-20140028) and the Data Protection Agency (S-20160164). Informed consent was obtained from all participants.

Provenance and peer review Not commissioned; externally peer reviewed.

Data sharing statement № additional data are available.

Open access This is an open access article distributed in accordance with the Creative Commons Attribution Non Commercial (CC BY-NC 4.0) license, which permits others to distribute, remix, adapt, build upon this work non-commercially, and license their derivative works on different terms, provided the original work is properly cited, appropriate credit is given, any changes made indicated, and the use is non-commercial. See: http://creativecommons.org/licenses/by-nc/4.0/. 


\section{REFERENCES}

1. Chugh SS, Havmoeller R, Narayanan K, et al. Worldwide epidemiology of atrial fibrillation: a global burden of disease 2010 study. Circulation 2014;129:837-47.

2. Krijthe BP, Kunst A, Benjamin EJ, et al. Projections on the number of individuals with atrial fibrillation in the European Union, from 2000 to 2060. Eur Heart J 2013;34:2746-51.

3. Freedman B, Camm J, Calkins $\mathrm{H}$, et al. Screening for atrial fibrillation: a report of the AF-SCREEN international collaboration. Circulation 2017;135:1851-67.

4. Mairesse GH, Moran P, Van Gelder IC, et al. Screening for atrial fibrillation: a European heart rhythm Association (EHRA) consensus document endorsed by the heart rhythm Society (HRS), Asia Pacific heart rhythm Society (APHRS), and Sociedad Latinoamericana de Estimulación Cardíaca Y Electrofisiología (SOLAECE). Europace 2017;19:1589-623.

5. Siontis KC, Gersh BJ, Killian JM, et al. Typical, atypical, and asymptomatic presentations of new-onset atrial fibrillation in the community: characteristics and prognostic implications. Heart Rhythm 2016;13:1418-24.

6. Kotecha D, Breithardt G, Camm AJ, et al. Integrating new approaches to atrial fibrillation management: the 6th AFNET/EHRA consensus Conference. Europace 2018;20:395-407.

7. Svennberg E, Engdahl J, Al-Khalili F, et al. Mass screening for untreated atrial fibrillation the STROKESTOP study. Circulation 2015;131:2176-84.

8. Gladstone DJ, Bui E, Fang J, et al. Potentially preventable strokes in high-risk patients with atrial fibrillation who are not adequately anticoagulated. Stroke 2009;40:235-40.

9. Kirchhof P, Benussi S, Kotecha D, et al. ESC guidelines for the management of atrial fibrillation developed in collaboration with EACTS. Eur Heart J 2016;2016:2893-962.

10. Vaes B, Stalpaert S, Tavernier K, et al. The diagnostic accuracy of the MyDiagnostick to detect atrial fibrillation in primary care. BMC Fam Pract 2014;15.

11. Hald J, Poulsen PB, Qvist I, et al. Opportunistic screening for atrial fibrillation in a real-life setting in general practice in Denmark-The atrial fibrillation found on routine detection (afford) non-interventional study. Plos One 2017;12:e0188086.

12. Wiesel J, Arbesfeld B, Schechter D. Comparison of the Microlife blood pressure monitor with the Omron blood pressure monitor for detecting atrial fibrillation. The American Journal of Cardiology 2014;114:1046-8.

13. Chan Pak-Hei, Wong Chun-Ka, Poh YC, et al. Diagnostic performance of a Smartphone-Based Photoplethysmographic application for atrial fibrillation screening in a primary care setting. $J$ Am Heart Assoc 2016;5.

14. Hobbs FDR, Fitzmaurice DA, Mant J, et al. A randomised controlled trial and cost-effectiveness study of systematic screening (targeted and total population screening) versus routine practice for the detection of atrial fibrillation in people aged 65 and over. the safe study. Health Technol Assess 2005;9:1-74.

15. Taggar JS, Coleman T, Lewis S, et al. Accuracy of methods for detecting an irregular pulse and suspected atrial fibrillation: a systematic review and meta-analysis. Eur J Prev Cardiolog 2016;23:1330-8.

16. Diederichsen ACP, Rasmussen LM, Søgaard R, et al. The Danish cardiovascular screening trial (DANCAVAS): study protocol for a randomized controlled trial. Trials 2015;16.

17. UNSCEAR. Report of the United Nations Scientific Committee on the Effects of Atomic Radiation to the General Assembly. In: Sources, Effects and Risks of lonizing Radiation, UNSCEAR 2012 Report. United Nations 2015:5-15.

18. Lowres N, Neubeck L, Salkeld G, et al. Feasibility and costeffectiveness of stroke prevention through community screening for atrial fibrillation using iPhone ECG in pharmacies. Thrombosis and Haemostasis 2014;111:1167-76.

19. Svennberg E, Stridh M, Engdahl J, et al. Safe automatic onelead electrocardiogram analysis in screening for atrial fibrillation. Europace 2017;19:1449-53.

20. Jahangir A, Lee V, Friedman PA, et al. Long-term progression and outcomes with aging in patients with lone atrial fibrillation. Circulation 2007;115:3050-6.

21. ClinicalTrials.gov. Systematic ECG Screening for Atrial Fibrillation Among 75 Year Old Subjects in the Region of Stockholm and Halland, Sweden - Full Text View. Available: https://clinicaltrials.gov/ ct2/show/NCT01593553 [Accessed 25 Mar 2018].

22. US Preventive Services Task Force, Curry SJ, Krist AH. et al. Screening for Atrial Fibrillation With Electrocardiography: US Preventive Services Task Force Recommendation Statement. JAMA 2018;320:478-84.

23. Camm AJ, Simantirakis E, Goette A, et al. Atrial high-rate episodes and stroke prevention. Europace 2017;19:169-79.

24. Mahajan R, Perera T, Elliott AD, et al. Subclinical device-detected atrial fibrillation and stroke risk: a systematic review and metaanalysis. Eur Heart J.

25. ClinicalTrials.gov. Apixaban for the Reduction of Thrombo-Embolism in Patients With Device-Detected Sub-Clinical Atrial Fibrillation - Full Text View. Available: https://clinicaltrials.gov/ct2/show/ NCT01938248 [Accessed 25 Mar 2018].

26. ClinicalTrials.gov. Non-vitamin K Antagonist Oral Anticoagulants in Patients With Atrial High Rate Episodes - Full Text View. Available: https://clinicaltrials.gov/ct2/show/NCT02618577 [Accessed 25 Mar 2018].

27. Lindholt JS, Søgaard R. Population screening and intervention for vascular disease in Danish men (VIVA): a randomised controlled trial. The Lancet 2017;390:2256-65. 